\title{
A percepção de agentes comunitárias de saúde sobre o planejamento reprodutivo com adolescentes
}

The perception of community health workers about reproductive life planning with adolescents

La percepción de las trabajadoras comunitarias de la salud sobre la planificación reproductiva con adolescentes

\section{Carolina Coutinho Costa ${ }^{\mathrm{I}}$, Camila Daiane Silva ${ }^{\mathrm{II}}$, Daniele Ferreira Acosta ${ }^{\mathrm{III}}$ Victoria Leslyê Rocha Gutmann ${ }^{\mathrm{IV}}$}

Resumo: Objetivo: conhecer a percepção de agentes comunitárias de saúde sobre o planejamento reprodutivo realizado com os adolescentes. Método: estudo qualitativo com dez agentes de uma unidade básica de saúde da família. Para a coleta de dados foi utilizado Grupo Focal. Utilizou-se a Análise de Conteúdo. Resultados: as agentes acreditavam na importância do planejamento reprodutivo com adolescentes, considerando o pouco diálogo intrafamiliar e a dificuldade em abordar o assunto nas visitas domiciliares. Reconheciam a necessidade de abordar o planejamento de modo ampliado, ao encontro do autoconhecimento, do respeito com o próximo e da prevenção das violências. Conclusão: a percepção das agentes pode ser favorecida ou prejudicada pela relação de proximidade com os profissionais de saúde. A temática deve ser discutida com os adolescentes, pois muitos não possuem comunicação com os pais, representando um tabu em muitos lares, seja por questões culturais ou por acreditarem ser um incentivo à prática sexual.

Descritores: Agentes comunitários de saúde; Adolescente; Planejamento familiar; Atenção primária à saúde; Saúde da família

Abstract: Objective: to know the community health workers' perception of reproductive life planning carried out with adolescents. Method: a qualitative study with ten agents from a Family Health Basic Unit. The Focal Group was used for data collection. Content Analysis was used. Results: the agents believed in the importance of reproductive life planning with adolescents, considering the little intra-family dialogue and the difficulty in approaching the subject during home visits. They recognized the need to approach reproductive planning in a broader way, meeting self-knowledge, respecting others, and preventing violence. Conclusion: the agents'

\footnotetext{
I Enfermeira. Especialista em Saúde da Família. Universidade Federal do Rio Grande. Rio Grande, Rio Grande do Sul, Brasil. coutinhocarolc@hotmail.com https://orcid.org/0000-0002-1093-5366

II Enfermeira. Doutora em Enfermagem. Universidade Federal do Rio Grande. Rio Grande, Rio Grande do Sul, Brasil. camilad.silva@yahoo.com.br https://orcid.org/0000-0002-0739-4984

III Enfermeira. Doutora em Enfermagem. Universidade Federal do Rio Grande. Rio Grande, Rio Grande do Sul, Brasil. nieleacosta@gmail.com. https://orcid.org/0000-0001-5690-1076

IV Enfermeira. Graduada em Enfermagem. Universidade Federal do Rio Grande. Rio Grande, Rio Grande do Sul, Brasil. victorialeslye@gmail.com https://orcid.org/0000-0002-3457-7620
} 
A percepção de agentes comunitárias de saúde sobre o planejamento reprodutivo com... $\mid 2$

perception can be favored or impaired by a close relationship with health professionals. The theme should be discussed with adolescents, as many have no communication with their parents, representing a taboo in many homes, either for cultural reasons or because they believe it is an incentive to sexual practice.

Descriptors: Community health workers; Adolescent; Family planning; Primary health care; Family Health

Resumen: Objetivo: conocer la percepción de las trabajadoras comunitarias de la salud sobre la planificación reproductiva realizada con adolescentes. Método: estudio cualitativo con diez trabajadoras de una unidad básica de salud familiar. El grupo focal se utilizó para la recopilación de datos. Se utilizó el análisis de contenido. Resultados: las trabajadoras creían en la importancia de la planificación reproductiva con los adolescentes, considerando la poca cantidad de diálogo intrafamiliar y la dificultad de abordar el tema durante las visitas domiciliarias. Reconocieron la necesidad de abordar la planificación de un modo más amplio, en búsqueda del autoconocimiento, del respeto por los demás y evitando la violencia. Conclusión: la estrecha relación con los profesionales de la salud puede favorecer o perjudicar la percepción de las trabajadoras. El tema debe discutirse con los adolescentes, ya que muchos no tienen comunicación con sus padres, lo que representa un tabú en muchos hogares, ya sea por razones culturales o porque creen que es un incentivo para la práctica sexual.

Descriptores: Agentes comunitarios de la salud; Adolescente; Planificación Familiar; Atención Primaria de Salud; Salud de la Familia

\section{Introdução}

A adolescência, compreendida entre a infância e a fase adulta, é marcada pela complexidade do crescimento, desenvolvimento biopsicossocial e amadurecimento. A Organização Mundial da Saúde (OMS) define a adolescência como a faixa etária entre 10 e 19 anos, jovens de 15 a 24 anos e adultos jovens de 20 a 24 anos. ${ }^{1}$ Já o Estatuto da Criança e do Adolescente (ECA) considera adolescente toda pessoa entre 12 e 18 anos e, expresso em lei, o Estatuto se aplica também àqueles entre 18 e 21 anos de idade. ${ }^{2}$ Nessa fase da vida as pessoas estão mais suscetíveis a diversas situações de maior ou menor vulnerabilidade social, individual ou coletiva. ${ }^{1}$

Nesse sentido, são frequentes a ambivalência de sentimentos, a insegurança, eclosão de dúvidas, a vergonha, somadas ao medo de rejeição pelo grupo. Tais aspectos fazem com o que o adolescente apresente dificuldades, ou sofra influência negativa, na tomada de decisão referente à própria saúde. A exemplo, destaca-se a dificuldade em negociar o uso de preservativo nas relações, aumentando o risco de Infecções Sexualmente Transmissíveis (ISTs) e de gestação não planejada. ${ }^{3}$ Para abordar tais situações, destaca-se o modelo de assistência da Estratégia de 
Saúde da Família (ESF), considerada porta de entrada para os outros serviços da rede. Equipes multidisciplinares trabalham com medidas de educação, de promoção e de prevenção de doenças e agravos, buscando atender às necessidades da população. Uma das ações realizadas pelos profissionais, incluindo os Agentes Comunitários de Saúde (ACS), é a condução de grupos específicos, tal como de hipertensos, de gestantes ou de adolescentes. ${ }^{4}$ Esses encontros permitem abordagens diferenciadas com vistas a promover a corresponsabilização do sujeito pelo seu cuidado.

No que se refere ao público adolescente, destaca-se a abordagem de questões que, na maioria das vezes, não são conversadas em casa ou aprofundadas na escola, por exemplo, a sexualidade, o uso de métodos contraceptivos ${ }^{1}$ e o projeto de vida. Dados epidemiológicos revelam tal necessidade, principalmente no que se refere ao planejamento reprodutivo entre adolescentes. No ano de 2018, 15,5\% das gestações registradas no Brasil eram de adolescentes com 10 a 19 anos. Em um município ao Sul do Brasil foi identificado que 13\% das gestantes possuíam entre 10 e 19 anos de idade e $24,1 \%$, de 20 a 24 anos. $^{5}$

Nesse sentido, destaca-se o papel do ACS, pois é o elo entre a comunidade e a equipe de saúde. ${ }^{6}$ Esses profissionais conhecem as dificuldades e os dilemas do dia a dia, pois moram na área de abrangência, o que contribui para a melhoria da atenção à saúde. A sua familiaridade no bairro, nas escolas, na unidade favorece a construção e a manutenção do vínculo com os adolescentes.

Dessa forma, é fundamental problematizar as percepções dos ACS sobre o planejamento reprodutivo $(\mathrm{PR})$ para adolescentes, considerando suas dúvidas, crenças, opiniões, de modo a fortalecer uma atuação efetiva e responsável ao atender as demandas deste grupo específico. A literatura revela que a falta de atividades de educação permanente e a fragilidade na interação com a equipe são problemas que afetam o trabalho sólido do agente, pois muitas vezes não está apto para abordar certos temas. ${ }^{7}$ 
A percepção de agentes comunitárias de saúde sobre o planejamento reprodutivo com... $\mid 4$

Diante de problemáticas tais como violência, pobreza, maus-tratos, situações de difícil abordagem entre pais e filhos, entre a escola e estudantes, o ACS tem papel primordial. São eles que, muitas vezes, conduzem o diálogo, a interlocução entre os atores, pois, sem que haja a construção de uma relação de confiança com a comunidade, não há como acessar as famílias e as residências. ${ }^{6}$

Assim, é ímpar problematizar com os ACS o que pensam sobre o planejamento reprodutivo para adolescentes. Acredita-se que conhecer suas percepções sobre o tema possibilitará fortalecer os saberes ao encontro de uma práxis que seja agradável para os agentes e atrativa para os adolescentes. Nesse sentido, este estudo tem como objetivo conhecer a percepção das agentes comunitárias de saúde sobre o planejamento reprodutivo realizado com os adolescentes.

\section{Método}

Trata-se de um estudo descritivo, exploratório, com abordagem qualitativa. Foi realizado por meio da técnica de Grupo Focal (GF), no período de setembro a novembro de 2017. Foram convidadas a participar do estudo todas as doze ACS que atuavam na Unidade Básica de Saúde da Família (UBSF) de um município ao extremo Sul do Brasil. A pesquisa foi realizada na unidade em que a Residência Multiprofissional em Saúde da Família estava lotada.

Participaram do GF dez agentes comunitárias de saúde, pois duas delas se encontravam em licença de saúde e férias no momento da coleta de dados. Destaca-se que a quantidade de participantes no GF é variável na literatura, recomendando de três a doze pessoas, a fim de obter melhor êxito dos resultados, não ter o risco de desviar o foco da temática e possibilitar que todos participem. ${ }^{8}$

Para a realização do GF, contou-se com a figura da moderadora, que neste caso era a residente de enfermagem, e apresentou as motivações e as razões para investigação do tema. 
5 | Costa CC, Silva CD, Acosta DF, Gutmann VLR

Após uma conversa introdutória, a moderadora instigou as falas das participantes por meio de um roteiro de questões norteadoras sobre o tema, facilitando trocas e mantendo os objetivos do trabalho do grupo. As questões norteadoras foram: Qual o entendimento do grupo sobre planejamento reprodutivo? Como vocês visualizam o planejamento reprodutivo no trabalho do ACS? Existem dificuldades e facilidades para abordar o tema? Qual o público-alvo do planejamento reprodutivo? Qual a importância de abordar o planejamento reprodutivo entre os adolescentes? Quais estratégias são necessárias para qualificar o planejamento reprodutivo entre os adolescentes?

Foram realizados três encontros com a presença de todas as dez ACS, com duração média de 90 minutos cada, na sala de reuniões da UBSF, nas quartas-feiras, dia em que ocorria o expediente interno da unidade. O primeiro encontro foi para apresentação do estudo, explanação sobre o Termo de Consentimento Livre e Esclarecido, bem como acerto para a data dos encontros futuros, enquanto os outros dois abordaram a temática. Optou-se por encerrar a pesquisa no terceiro encontro, considerando a repetição de informações e a saturação dos dados. Após o fim de cada encontro, a moderadora realizou anotações acerca das impressões das participantes, das reações e de manifestações contraditórias. Durante a coleta estiveram presentes na sala apenas os participantes e a pesquisadora. Utilizou-se, a cada encontro, o suporte de gravador digital para a posterior transcrição dos áudios.

Como técnica de análise de dados foi realizada a Análise de Conteúdo, proposta por Bardin, que propõe três etapas - exploração do material, tratamento dos resultados e interpretação. A primeira contou com a organização do material, na qual se fez a leitura minuciosa dos resultados, buscando particularidades de cada informante para a elaboração das ideias e possíveis categorias. Após essa busca, a autora realizou a codificação de temas, de forma que pudessem ser agrupados em núcleos semelhantes que, por fim, deram origem às categorias. ${ }^{9}$ 
A percepção de agentes comunitárias de saúde sobre o planejamento reprodutivo com... | 6

Para manter o anonimato das participantes, elas foram identificadas pelas iniciais ACS seguidas da numeração sucessiva (ACS1, ACS2...). O projeto foi aprovado em abril de 2017, pelo Comitê de Ética em Pesquisa sob CAAE nº 66563217.4.0000.5324.

\section{Resultados}

As dez mulheres participantes atuavam na unidade entre cinco e dezesseis anos. A idade mínima foi de 29 anos e a máxima de 52. Sobre a situação conjugal, eram metade casadas e metade solteiras, e todas tinham filhos. Apenas uma ACS tinha ensino médio incompleto e o restante, ensino médio completo. A maioria delas mencionou ter participado de alguma capacitação em planejamento reprodutivo.

As falas das ACS abordaram como principais temáticas a visão geral sobre os adolescentes, a perspectiva de um PR ampliado, a necessidade de abordar a sexualidade nesta etapa da vida devido à falta de comunicação com os pais, bem como pelas dúvidas e tabus que permeiam esta fase. Além disso, constataram-se as facilidades e dificuldades em discutir o tema em pauta com os adolescentes, sendo o vínculo e a proximidade com as famílias o motivo catalisador em ambas.

Em relação ao grupo etário, as ACS viam os adolescentes como vulneráveis, desinteressados por sua saúde e irresponsáveis.

Adolescência para mim é a pior parte da vida, porque é a transformação, eles não escutam pai, amigos, eles escutam aquilo que querem ouvir. Eu vou fazer aquilo que eu quero. Os adolescentes precisam de ajuda de vários lados. (ACS10)

Acho que tem muita falta de interesse e falta de cuidado, porque falta de informação não é [...], mas eu acho que falta de informação de jeito nenhum não é, é falta de interesse mesmo da pessoa [...], e aquele negócio: 'não vai dar nada' e acaba dando. (ACS7) 
Eu vejo que eles querem ter liberdade, mas não querem ter compromisso. (ACS1)

Por outro lado, elas reconheciam a importância de abordar o planejamento reprodutivo com adolescentes, destacando a necessidade de problematizar aspectos que vão além da sexualidade, ao encontro do cuidado com o corpo e com o próximo.

A orientação sexual vai além das doenças, é uma questão de orientar respeito como um todo, conhecer seu corpo, respeitar o outro [...]. Então, acho que não tem que trabalhar só as doenças, tem que trabalhar todo o cuidado consigo mesmo. (ACS8)

Até mesmo porque eles se batem, se agridem, a violência está exposta também, não só a sexualidade, tu vês meninos agredindo as meninas e elas também agredindo os meninos. Então assim, o assunto é bem complexo, o planejamento reprodutivo abrange muita coisa. (ACS4)

A conversa com eles é diferente, a gente não pensa só no método anticoncepcional, essas coisas assim com eles, a gente fala sobre o cuidado. $O$ mais importante, para mim, do que ela tomar um anticoncepcional, é fazer uso do preservativo. (ACS5)

As participantes também destacaram que nesta idade há falta de diálogo entre os adolescentes e os pais, fato que compromete os seus cuidados em saúde. No presente estudo, ficou evidente que a falha na comunicação também estava relacionada às questões culturais.

A sexualidade na adolescência é muito cultural, porque, por mais que tenham uma orientação de nós, se não tiver uma boa base familiar, com exemplos, uma conversa aberta, eu acredito que não adianta muito, eles não respondem a isso. Eles fazem o que eles querem, conforme a base familiar que eles têm. (ACS 8) 
A percepção de agentes comunitárias de saúde sobre o planejamento reprodutivo com... $\mid 8$

Nesse sentido, reforçaram a necessidade de acompanharem esse grupo específico fosse na unidade, com outros profissionais, fosse por meio de mensagem ou WhatsApp. Acreditavam que as questões relacionadas à sexualidade e à reprodução precisam ser problematizadas, considerando ser uma fase permeada por dúvidas e tabus.

Uma coisa que ajuda muito é a Internet, eu já recebi mensagem de uma menina: "Desculpa te incomodar, mas eu acho que estou grávida, mas não fala para ninguém, me ajuda...”[...] Mas tudo era por mensagem, se ela me via, ficava com vergonha. (ACS4)

Eles não têm liberdade para sentar e conversar com os pais e perguntar, então, às vezes é mais fácil vir numa consulta de enfermagem e tirar todas as dúvidas. (ACS7)

Ou então a mãe deixa aquele tabu: 'Não posso ficar falando de sexo, senão vou estar incentivando a pensar nisso.’. (ACS 4)

Diante dos entraves que permeiam o planejamento reprodutivo na adolescência, as ACS acreditavam que poderiam contribuir com ações de cuidado. Além disso, conhecer os pais e o contexto de vida dos adolescentes faz a diferença na atenção à saúde.

Dentro do trabalho já é automático perguntar sobre isso [planejamento reprodutivo]. Vemos uma mãe que conhecemos e conhecemos também a adolescente, sabemos como ela é na rua, na escola, então sabemos como abordar qualquer pessoa da família que tenhamos um relacionamento. Então, essa orientação é o olhar, o nosso olhar dentro do domicílio da família que faz a diferença. (ACS10)

Todavia, ao mesmo tempo em que o vínculo das ACS com as famílias é algo positivo, também dificulta o diálogo das adolescentes com essas profissionais. Isso ocorre, pois acreditam que as informações serão reveladas aos seus pais. 
9 | Costa CC, Silva CD, Acosta DF, Gutmann VLR

Muitos nem perguntam para nós, com medo que falemos alguma coisa para família, para a mãe. (ACS5)

No início é aquela coisa complicada, nem nos conhecíamos. Mas assim: ' $O$ que precisar pode falar comigo, não vamos falar para o pai, não vamos falar para a mãe, nós estamos aqui para te ajudar’. (ACS10)

No mesmo sentido, as agentes referiram que sua atuação junto aos adolescentes poderia ficar prejudicada pelo fato de que as visitas domiciliares eram realizadas dentro da própria casa desses indivíduos, muitas vezes na presença dos pais, irmãos e familiares que moravam junto, impedindo uma conversa mais sigilosa.

A fala com os adolescentes no domicílio é complicada, porque sentamos ali, olhamos, queremos falar sobre o assunto, mas já te cortam, ou na frente da mãe ou do pai eles ficam mais inibidos. Então, eu digo que santo de casa não faz milagre, porque nós já somos da área. (ACS10)

A gente consegue acessar aquele adolescente? Sim, mas dificilmente aquele adolescente ou aquela adolescente que de repente já está começando a sua vida sexual, ou pensando em começar a sua vida sexual, vai perguntar qualquer dúvida para nós na visita da família. (ACS5)

Com isso, percebe-se que, ao mesmo tempo em que o vínculo com as famílias facilita orientar o adolescente, também dificulta a aproximação espontânea desse jovem com o profissional.

\section{Discussão}

As ACS relataram que a adolescência é uma fase na qual os jovens negam a orientação dos pais, são irresponsáveis, não planejam suas ações, são inconsequentes e só prestam atenção na opinião do próprio grupo. A literatura nos mostra que os adolescentes têm um afastamento 
A percepção de agentes comunitárias de saúde sobre o planejamento reprodutivo com... $\mid 10$

dos seus familiares e a inserção em grupos de pessoas com a mesma idade, sendo a tecnologia um fator de grande influência nesse processo. ${ }^{10}$

Diante do despreparo deste grupo para o planejamento reprodutivo, é preciso abordar uma visão ampliada com abordagem simultânea de outras temáticas. Diante disso, as ACS compreenderam outras facetas do PR, voltadas ao autoconhecimento, ao respeito ao próximo, à prevenção de violências e à cultura da paz. É preciso aproveitar todas as oportunidades de encontro com adolescentes para trabalhar o projeto de vida, fazendo-os os repensar sobre a reponsabilidade de ser pai e mãe. A violência simbólica, atualmente, tem permeado a vida de casais jovens ${ }^{11}$ e, portanto, é pertinente e faz parte da abordagem da temática.

Por parte dos adolescentes, a literatura ${ }^{12}$ apresenta que eles não conseguem tirar dúvidas com os pais, seja porque a família não problematiza o assunto ou porque não estão disponíveis para conversa num momento satisfatório. ${ }^{12}$ Diante disso, a consulta de enfermagem foi referida como recurso adicional nesse processo de educação sexual, tendo em vista que os adolescentes estão em uma fase em que necessitam de atenção especial. Nesse sentido, o planejamento reprodutivo oferecido pelo serviço de saúde é fundamental, sendo o ACS o profissional que participa ativamente deste cenário.

As ACS também referiram que os adolescentes têm pouco diálogo com suas famílias sobre o assunto sexualidade. Nesse sentido, alguns autores apregoam que as famílias têm a intenção de proteger os adolescentes, dificultando a fala sobre a educação sexual, pois acreditam que problematizar o assunto influencia a prática sexual precocemente, ${ }^{13}$ tal como evidenciado.

Outras vezes, há vergonha por parte dos pais, que cortam o assunto sem atribuir importância às opiniões do adolescente. ${ }^{12}$ Isso pode ocorrer devido à mudança de fase dos filhos, que antes eram crianças e agora são adolescentes cheios de dúvidas e, assim como eles estão se transformando e não sabem lidar com os problemas que surgem, os pais também estão nessa fase de mudança da maneira de criar seus filhos. 
Algo que facilita a comunicação e possibilitaria uma aproximação maior entre ACS e adolescentes é o uso de chats de mensagem, pois assim eles não precisam se deparar com outra pessoa, sentindo-se mais motivados para expressarem seus sentimentos e sanar dúvidas. As mídias estão à disposição a qualquer momento, facilitando a busca por respostas imediatas diante de suas necessidades. ${ }^{12}$

Observa-se que a proximidade com as famílias facilita a visão sobre o contexto de vida, propiciando uma abordagem holística. Elas estabelecem diálogos, desenvolvem relações de afeto, problematizam as situações e constroem, de forma compartilhada, o conhecimento, permitindo a livre expressão e a valorização dos sujeitos na sua realidade. ${ }^{14}$

Conhecer as famílias era relevante para as ACS, pois sabe-se que as crenças familiares muitas vezes são passadas de geração em geração, favorecendo a abordagem pelo profissional de saúde. Ao mesmo tempo, muitas vezes, é difícil desmistificar certas condutas, conforme evidenciado em estudo que observou mães adolescentes reproduzindo, no cotidiano de cuidado do bebê, várias práticas que faziam parte da cultura local e familiar, tais como não oferecer queijo, acreditando que pode prejudicar o desenvolvimento da fala, utilização de chás para determinadas enfermidades, sem o devido embasamento científico. ${ }^{15}$ As ACS, por residirem no mesmo bairro, muitas vezes conhecem tais crenças, facilitando a problematização sobre os benefícios e malefícios.

O fortalecimento do vínculo possibilita que o agente seja a referência na unidade de saúde, bem como nos espaços de atuação ao entorno da comunidade, sendo vistos de maneira positiva por parte dos adolescentes. A comunicação entre ACS e adolescentes pode ficar prejudicada devido ao receio de que eles contem aos responsáveis sobre aquilo que foi discutido, e por vezes isso se configura como empecilho na obtenção do vínculo com as ACS e na adesão às ações voltadas ao planejamento reprodutivo. Por mais que esse medo exista entre os adolescentes, é necessário que os ACS sejam multiplicadores das informações, garantindo o 
A percepção de agentes comunitárias de saúde sobre o planejamento reprodutivo com... $\mid 12$

sigilo profissional independentemente da idade, desde que não haja risco de vida ou risco relevante a terceiros. ${ }^{1}$

Por outro lado, mesmo que esse direito seja garantido, há uma falha na capacitação dos profissionais para atender esse público. ${ }^{16}$ Logo, muitas vezes, limitam-se em procurar auxílio nas unidades básicas de saúde por não se sentirem compreendidos pelos profissionais. Além disso, o fato de os ACS fazerem suas visitas domiciliares na casa onde moram os adolescentes parece tornar-se um impedimento para uma conversa mais eficiente.

Ainda que na literatura esse fato não apareça como um agravante, percebeu-se, neste estudo, como um aspecto que prejudica na promoção de ações produtivas com esses adolescentes. O trabalho dos ACS é fundamental dentro da comunidade, pois eles moram no mesmo bairro em que atuam, fazendo com que eles vivenciem as mesmas dificuldades dos usuários, identificando as prioridades que precisam ser resolvidas. ${ }^{6}$ Portanto, é preciso problematizar as facilidades e dificuldades no processo de trabalho dos ACS, a fim de qualificar as ações em saúde, minimizando as barreiras que impedem uma abordagem efetiva ao encontro das necessidades dos adolescentes.

\section{Conclusão}

A percepção das ACS sobre o planejamento reprodutivo realizado com os adolescentes é de que se trata de uma atividade relevante, podendo ser favorecida ou prejudicada pela relação de proximidade das profissionais de saúde com as famílias dos adolescentes. Por um lado, os adolescentes podem se sentir confortáveis por conhecerem a profissional de saúde, por outro, podem suspeitar que ela irá expor as dúvidas aos pais. Dessa forma, perceberam que a visita domiciliar se torna um momento inadequado para conversar com esses jovens, considerando que a presença de familiares os inibe. 
As ACS tinham a percepção de que o planejamento reprodutivo com os adolescentes é necessário, pois muitos não possuem comunicação com os pais sobre a temática, a qual representa um tabu em muitos lares, seja pela questão cultural ou por acreditarem que o diálogo sobre o tema se tornará um incentivador da prática sexual. Por outro lado, as agentes comunitárias de saúde perceberam que o adolescente é pouco interessado pela própria saúde, necessitando de métodos atrativos e cativantes, como mensagens por aplicativos.

No que se refere às limitações do estudo, aponta-se o número reduzido de participantes, o que torna difícil as generalizações a partir dos dados, indicando a necessidade de ampliar para outras unidades de saúde, inclusive de municípios diferentes. Os resultados deste estudo podem contribuir para as áreas da saúde e enfermagem, evidenciando a percepção dos agentes comunitárias de saúde, como componentes da equipe, atuando no planejamento reprodutivo dos adolescentes, buscando minimizar as dificuldades na atuação dos ACS e fortalecer as práticas de sucesso com o grupo.

\section{Referências}

1. Ministério da Saúde (BR). Marco Legal: saúde, um direito de adolescentes [Internet]. Brasília (DF): Ministério da Saúde; 2007 [acesso em 2019 jul 03]. Disponível em: http://bvsms.saude.gov.br/bvs/publicacoes/07_0400_M.pdf

2. Centro de Defesa dos Direitos da Criança e do Adolescente (CEDECA). Estatuto da Criança e do Adolescente [Internet]. Rio de Janeiro (RJ): CEDECA; 2017 [acesso em 2019 jul 03]. Disponível em: https://cedecarj.org.br/portfolio/estatuto-da-crianca-e-do-adolescente-2017/

3. Silva MRB, Silva LA, Maturana HCA, Silva RB, Santos ME, Figueiredo Filho V. Por que elas não usam? um estudo sobre a não adesão das adolescentes ao preservativo e suas repercussões. Saúde Redes. 2015;(1)4:75-83. doi: https://doi.org/10.18310/2446-4813.2015v1n4p75-83

4. Dorneles DF, Hatzenberger DHC, Schnorr L. Percepção dos usuários sobre os grupos e espaços de vivência e convivência no Bairro Lomba do Pinheiro em Porto Alegre. Saúde Redes [Internet]. 2019 [acesso em 2019 jul 03];(5)1:75-104. Disponível em: http://revista.redeunida.org.br/ojs/index.php/redeunida/article/view/982 
A percepção de agentes comunitárias de saúde sobre o planejamento reprodutivo com... $\mid 14$

5. Ministério da Saúde (BR). Datasus. TABNET. Estatísticas vitais [Internet]. 2018 [acesso em 2020 abr 24]. Disponível em: http://www2.datasus.gov.br/DATASUS/index.php?area=0205

6. Alonso CMC, Béguin PD, Duarte FJCM. Trabalho dos agentes comunitários de saúde na estratégia saúde da família: metassíntese. Rev Saúde Pública. 2018;(52)14:1-13. doi: https://doi.org/10.11606/S15188787.2018052000395

7. Almeida AM, Machado BR, Silva FMR, Quadros KAN. Dificuldade dos agentes comunitários de saúde na prática diária. Rev Med Minas Gerais [Internet]. 2016 [acesso e 2019 ago 24];26:e-1800. Disponível em: http://rmmg.org/artigo/detalhes/2081

8. Souza LK. Recomendações para a realização de grupos focais na pesquisa qualitativa. Psi UNISC. 2020;(4)1:52-66. doi: http://dx.doi.org/10.17058/psiunisc.v4i1.13500

9. Bardin L. Análise de conteúdo. Lisboa: Edições 70; 2011.

10. Neumann DMC, Missel RJ. Família digital: a influência das tecnologias nas relações entre pais e filhos adolescentes. Pensando Fam [Internet]. 2019 [acesso em 2020 abr 15];(23)2:75-91. Disponível em: http://pepsic.bvsalud.org/scielo.php?script=sci_arttext\&pid=S1679-

494X2019000200007\&lng=pt\&nrm=iso\&tlng=pt

11. Bittar DB, Nakano AMS. Violência simbólica entre adolescentes nas relações afetivas do namoro. Rev Esc Enferm USP. 2017:(51)e:03298. doi: http://dx.doi.org/10.1590/S1980-220X2017003003298

12. Aragão JMN, Gubert FA, Torres RAM, Silva ASR, Vieira NFC. O uso do facebook na aprendizagem em saúde: percepções dos adolescentes escolares. Rev Bras Enferm. 2018;(17)2:286-92. doi: https://doi.org/10.1590/0034-7167-2016-0604

13. Queiroz MVO, Vasconcelos MM, Alcântara CM, Fé MCM, Silva ANS. Características sociodemográficas e gineco-obstétricas de adolescentes assistidas em serviço de planejamento familiar. Rev Enferm UFSM. 2017;(7)4:615-28. doi: http://dx.doi.org/10.5902/2179769226988

14. Maciazeki-Gomes RC, Souza CD, Baggio L, Wachs F. O trabalho do agente comunitário de saúde na perspectiva da educação popular em saúde: possibilidades e desafios. Ciênc Saúde Colet. 2016;(21)5:163746. doi: https://doi.org/10.1590/1413-81232015215.17112015

15. Cuellar KJM, Vargas EC. Creencias y prácticas culturales de las madres sobre el cuidado del niño menor de cinco años em Tarata-Tacna. Rev Méd Basadrina [Internet]. 2019 [acceso en 2020 abr 24];(13)2:71-78. Disponible en: http://www.revistas.unjbg.edu.pe/index.php/rmb/article/view/884/950

16. Netto JJM, Dias MSA, Machado MFAS, Gubert FA, Vasconcelos MIO, Oliveira MS, et al. Construção e validação de instrumento para subsidiar o cuidado ao adolescente na Atenção Primária à Saúde. Adolesc Saúde [Internet]. 2018 [acesso em 2019 ago 24];(15)2:92-101. Disponível em: http://www.adolescenciaesaude.com/detalhe_artigo.asp?id=724 
15 | Costa CC, Silva CD, Acosta DF, Gutmann VLR

\section{Autor correspondente}

Carolina Coutinho Costa

E-mail: coutinhocarolc@hotmail.com

Endereço: General Osório, s/n, Bairro Centro, Rio Grande/RS

CEP: $96200-400$

\section{Contribuições de Autoria}

\section{1 - Carolina Coutinho Costa}

Concepção, planejamento do projeto de pesquisa, obtenção, análise e interpretação dos dados, redação e revisão crítica. Autora do Trabalho de Conclusão da Residência do programa de Residência Multiprofissional em Saúde da Família intitulado: Planejamento Familiar para adolescentes: a percepção do agente comunitário de saúde, que originou este artigo.

\section{2 - Camila Daiane Silva}

Interpretação dos dados, redação e revisão crítica.

\section{3 - Daniele Ferreira Acosta}

Concepção, planejamento do projeto de pesquisa, obtenção, análise e interpretação dos dados, redação e revisão crítica.

\section{4 - Victoria Leslyê Rocha Gutmann}

Redação e revisão crítica.

\section{Como citar este artigo}

Costa CC, Silva CD, Acosta DF, Gutmann VLR. A percepção de agentes comunitárias de saúde sobre o planejamento reprodutivo com adolescentes. Rev. Enferm. UFSM. 2020 [Acesso em: Anos Mês Dia]; vol.10 e57: 1-15. DOI:https://doi.org/10.5902/2179769240345 